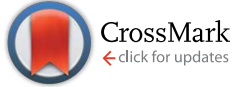

Cite this: RSC Adv., 2016, 6, 16868

Received 10th December 2015 Accepted 2nd February 2016

DOI: $10.1039 / c 5 \mathrm{ra} 26377 \mathrm{~h}$

www.rsc.org/advances

\section{Entrapment and characterization of functional allosteric conformers of hemocyanin in sol-gel matrices $\uparrow$}

\author{
Fabrizio Minute, ${ }^{a}$ Nadja Hellmann, ${ }^{\text {b }}$ Francesco Spinozzi, ${ }^{\mathrm{c}}$ Maria Grazia Ortore, ${ }^{\mathrm{c}}$ \\ Paolo Di Muro, ${ }^{a}$ Luigi Bubacco ${ }^{a}$ and Mariano Beltramini ${ }^{\star a}$
}

Hemocyanins are giant oxygen transport proteins of molluscs and arthropods, which display high cooperativity and a complex pattern of conformations, generated by hierarchical allosteric interactions of their complex quaternary structure. A still unanswered question is the correlation between the functional properties of the postulated conformers and structural features that govern their oxygen binding, such as metal complex coordination. In this study we focus on the dodecameric hemocyanin of the crustacean Carcinus aestuarii, with the aim to obtain a functional and structural characterization of the individual conformational states giving rise to cooperativity, by entrapping hemocyanin into a sol-gel matrix. The latter has attracted much attention as an ideal substrate for immobilization of macromolecules within the pores of a hydrated and optically transparent matrix that preserves the structures and functionalities of the encapsulated macromolecules. In our experimental approach, the sol-gel is capable of blocking the conformational transitions of the hemocyanin induced by changing the oxygen concentration in solution studies. This enables characterization of both the oxygenated and deoxygenated forms of particular conformers. Here we describe the oxygen binding properties of individual matrix entrapped conformers of $C$. aestuarii hemocyanins and the spectroscopic features characteristic for these conformations. Since the quaternary structure itself is not altered, as the SANS data unambiguously show in the sol-gel a dodecameric organization for $C$. aestuarii hemocyanin, we propose that the entrapment of hemocyanin in a sol-gel is able to freeze functionally relevant conformational distributions under appropriate conditions. The spectroscopic characterization of the functionally characterized conformers trapped in the sol gel allowed us to assign the differences in the active site geometry as observed by XAS to individual conformations.

\section{Introduction}

An important aspect of functional control in biological macromolecules is the capacity to undergo structural changes in response to diverse environmental conditions. In allosteric proteins, the functional properties are modulated by allosteric effectors, which modify the binding equilibria with ligands/ substrates and the interactions among different domains of the molecule. ${ }^{1}$

Hemoglobin has been the paradigm for the investigation of allostery and cooperativity in macromolecules. In accordance

\footnotetext{
${ }^{a}$ Department of Biology, University of Padova, Via Ugo Bassi 58B, I-35131 Padova, Italy.E-mail: mariano.beltramini@unipd.it

${ }^{b}$ Institute for Molecular Biophysics, University of Mainz, Jakob-Welder-Weg, Mainz, Germany

${ }^{c}$ Department DISVA, Marche Polytechnic University and CNISM, Via Brecce Bianche, I60131 Ancona, Italy

$\dagger$ Electronic supplementary information (ESI) available: Four additional sections, eight tables and seven figures. See DOI: 10.1039/c5ra26377h
}

with the two-state Monod-Wyman-Changeaux (MWC) allosteric model, ${ }^{2}$ X-ray structures of fully oxygenated and fully deoxygenated hemoglobin confirmed the presence of two distinct structures for the tetramers, identified as the structure of the high affinity (R-state) and low-affinity states (T-state). ${ }^{3}$ The model postulates a non-cooperative oxygen binding in the absence of conformational transitions. Coherently, oxygenbinding curves of pure $\mathrm{T}$ and $\mathrm{R}$ states crystals ${ }^{4}$ exhibit noncooperative oxygen binding. These results were also confirmed through the encapsulation of hemoglobin into a silica gel matrix, circumventing the major criticism of a non-cooperative binding due to crystal lattice constraints. ${ }^{5-7}$

Hemocyanins, which are giant oxygen transport proteins of molluscs and arthropods, often display high cooperativity and a complex pattern of conformations, generated by hierarchical allosteric interactions within their complex quaternary structure. ${ }^{8}$ Due to the hierarchy of the quaternary structure, the oxygen binding equilibrium of hemocyanins can usually not be described simply by the MWC model but requires extensions such as the three-state model ${ }^{9-11}$ or hierarchical constructs of the 
MWC model where "nested" oligomers are considered. ${ }^{12,13}$ An unanswered question is the correlation between the functional properties of the postulated conformers and structural features that govern their oxygen binding, such as metal complex coordination geometry and tertiary/quaternary structure.

The active site of hemocyanins is a binuclear center, where the molecular oxygen binds in a $\mu-\eta^{2}: \eta^{2}$ conformation as a peroxo bridge between the two copper ions. The quaternary structure is species-dependent, is only weakly related to the magnitude of cooperativity, as evaluated by the value of the Hillcoefficient $n_{\mathrm{H}}$, and is not related to the mean oxygen binding affinity $\left(p_{50}\right)$. Detailed structural information on the active site of hemocyanin became first available with the publication of the X-ray crystal structure of hexameric hemocyanin from Panulirus interruptus and Limulus polyphemus hemocyanins. ${ }^{\mathbf{1 4 - 1 6}} \mathrm{Six}$ histidines coordinate two copper ions ( $\mathrm{N} \varepsilon-\mathrm{Cu})$. In the deoxygenated state, the binuclear site contains $\mathrm{Cu}(\mathrm{I})$ and it is devoid of spectroscopic features above $300 \mathrm{~nm}$. The oxygenated form presents a binuclear $\mathrm{Cu}(\mathrm{II})-\mathrm{O}_{2}{ }^{2-}-\mathrm{Cu}(\mathrm{II})$ complex exhibiting characteristic charge transfer bands $\pi \sigma^{*} \rightarrow \mathrm{Cu}(\mathrm{II})$ and $\pi \mathrm{v}^{*} \rightarrow$ $\mathrm{Cu}(\mathrm{II})$ with $\lambda_{\max } \sim 345 \mathrm{~nm}\left(\varepsilon \sim 20000 \mathrm{M}^{-1} \mathrm{~cm}^{-1}\right) .{ }^{17-19}$ The absorbance at this wavelength is linearly related to the fraction of oxygenated hemocyanin. The coordination geometry of the active site was deduced from the features in the X-ray absorption near edge structure region (XANES) at the $\mathrm{Cu}$ K-edge of Xray absorption spectrum (XAS). In particular, the intensity of the transition at $8983 \mathrm{eV}$ has been qualitatively correlated to the displacement of copper from the equatorial plane defined by nitrogen ligands of the histidines. ${ }^{17,20,21} \mathrm{~A} \mathrm{pH}$-dependent change in the geometry of the active site for the deoxygenated hemocyanin was demonstrated using this technique, suggesting a structural basis for the Bohr effect. ${ }^{22}$ Conversely, L-lactate affects oxygen affinity by modulating the quaternary structure of hemocyanin, without modifications of the coordination geometry of the active site. ${ }^{23}$

In this work we focus on the hemocyanin of the crustacean Carcinus aestuarii, a typical example of $2 \times 6$ oligomeric crustacean hemocyanin..$^{\mathbf{2 4 , 2 5}}$ The aim is the functional and structural characterization of the individual conformational states giving rise to cooperativity by entrapping hemocyanin into a sol-gel matrix. In the last years, sol-gel material has attracted much attention as ideal substrate for immobilization of different biological materials such as organic molecules, proteins and cells, within the pores of an inert, hydrated and optically transparent matrix that preserves structures and functionalities of encapsulated specimens. ${ }^{26-30}$ Because of the porous nature of the sol-gel network (diameter pores of $\sim 2-100 \AA$ ), small ions or molecules can diffuse through the matrix to the site of entrapped proteins, producing a signal detectable by an appropriate sensing system. This feature is the key point for the development of biosensors based on sol-gel matrix.

The sol-gel technique represents a crucial tool for structural and functional characterization of allosteric proteins, through the ability of the matrix to strongly reduce conformational transitions. Steric restraints and specific electrostatic interactions between the entrapped proteins and the silica walls are implicated in the reduction of the large-scale dynamics of the entrapped biomolecules. ${ }^{5,6,31}$ Indeed, as reported for hemoglobin, ${ }^{32-34}$ the silica matrix slows down or eliminates the tertiary and quaternary relaxation of the protein, without affecting the equilibrium properties of ligand and effector binding for a given state. The entrapment of different conformational states in a sol-gel matrix was previously described for the large 24-meric Eurypelma californicum hemocyanin. ${ }^{35}$ Furthermore, it was utilized to develop a biocompatible oxygen sensor based on spectroscopic differences between oxygenated and deoxygenated hemocyanin. ${ }^{36}$ Here we describe the oxygen binding properties of the matrix entrapped individual conformers of $C$. aestuarii hemocyanins and also spectroscopic features characteristic for these conformations.

\section{Results}

A central part of the study was the encapsulation of hemocyanin in a sol-gel made of tetramethyl orthosilicate. To this end, sample holders were designed to ensure the maintenance of the bulk water and the presence of a sufficiently large surface to guarantee fast exchange of the solutes (see ESI $\dagger$ for details). To find the optimal buffer conditions for encapsulation of hemocyanin, the microstructure of the sol-gel matrix was analyzed at different $\mathrm{pH}$-values $(6.5,7.5$ and 7.8), in absence and presence of $25 \%$ glycerol, and at different times after the formation of the gel ( 2 and 6 days). The optimal gel should have high optical quality, in consideration of its use for spectroscopic analysis, and pores with dimension close to the size of the hemocyanin dodecamer. The optical properties of the sol-gel were improved in the presence of glycerol but did not depend on $\mathrm{pH}$, presence of L-lactate or aging time (see ESI $\dagger$ for details). Thus, the same protocol for encapsulation of hemocyanin was applied in all cases, employing inclusion of glycerol and aging time of 2 days. Once embedded into the sol-gel matrix no release of dodecameric hemocyanin into the bulk solution was observed within the time span of the oxygen binding experiments (hours).

\section{Oxygen binding properties of Carcinus aestuarii hemocyanin in solution and embedded in silica gel, the effect of $\mathrm{pH}$ and $\mathrm{L}^{-}$ lactate}

Oxygen binding curves were determined for three types of samples (solution, Deoxy ${ }^{\mathrm{EM}}$, Oxy ${ }^{\mathrm{EM}}$ ), under five different conditions. The conformers obtained by encapsulation of hemocyanin in absence and presence of 750-760 Torr oxygen are referred to as Deoxy $^{\mathrm{EM}}$ and $\mathrm{Oxy}^{\mathrm{EM}}$, respectively. Entrapped conformers studied at maximal oxygen partial pressure (750-760 Torr) are denoted $\operatorname{Deoxy}_{760}^{\mathrm{EM}}$ and $\mathrm{Oxy}_{760}^{\mathrm{EM}}$ and those investigated in absence of oxygen are denoted Deoxy ${ }_{0}^{\mathrm{EM}}$ and $\mathrm{Oxy}_{0}^{\mathrm{EM}}$. In all but one (Deoxy $\mathrm{E}_{760}^{\mathrm{EM}}$ at $\mathrm{pH}$ 6.5) the presence of 750 Torr oxygen leads to full oxygenation. Hemocyanin was entrapped at the same $\mathrm{pH}$ as intended for the oxygen binding experiments. Data are shown in the Hill-plot representation to allow visual inspection of cooperativity (slope around $\left.p_{50}\right)$ and the initial and final slopes to reveal the consequence of entrapment of hemocyanin (Fig. 1A-E).

As previously reported, ${ }^{24}$ binding curves of hemocyanin in solution from $C$. aestuarii show a typical Bohr-effect, leading to 

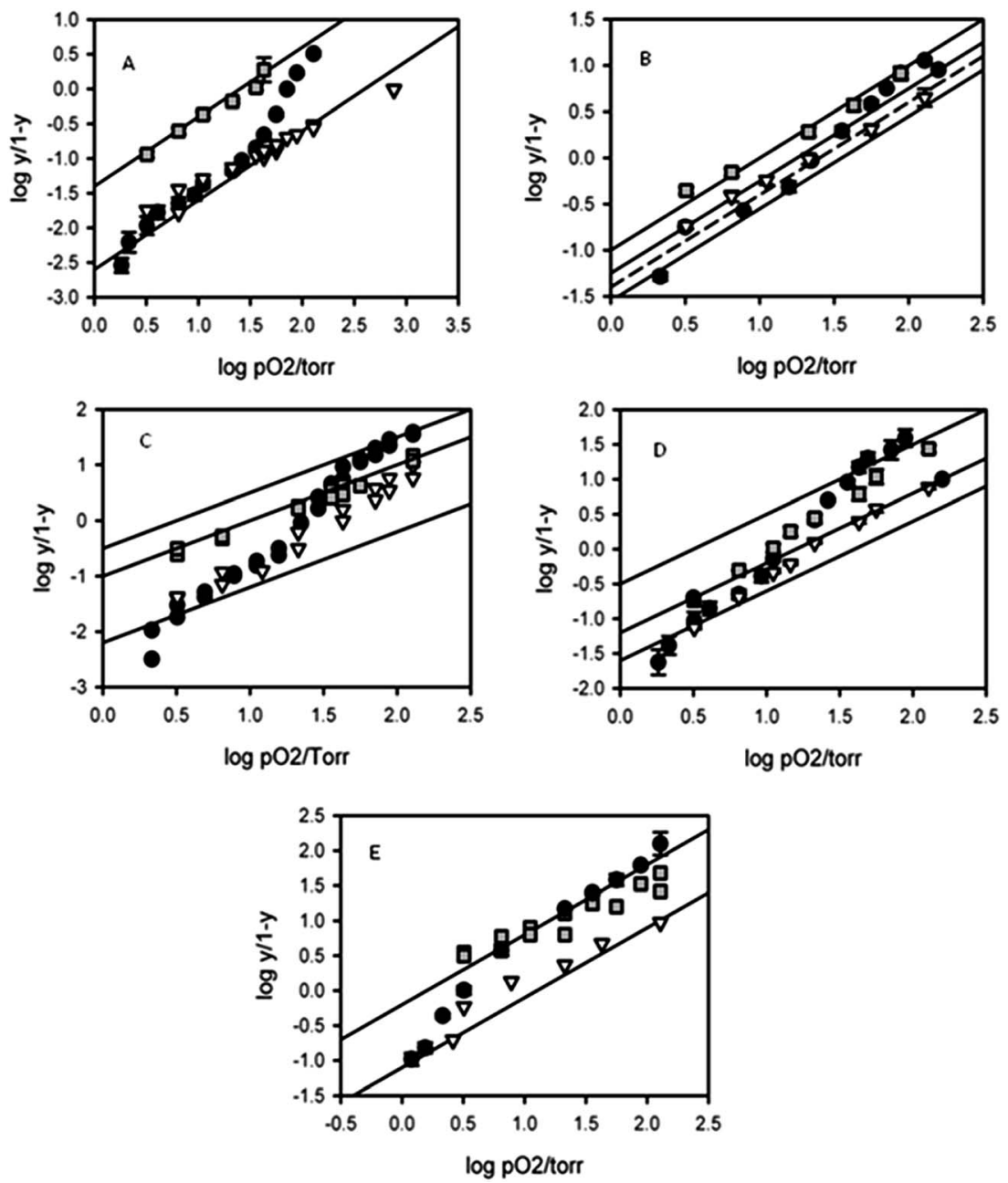

Fig. 1 Oxygen binding properties of Carcinus aestuarii hemocyanin. Hill-plots of hemocyanin in solution (filled circles), entrapped in absence of oxygen (Deoxy ${ }^{\mathrm{EM}}$, open triangles) and in presence of oxygen (Oxy ${ }^{\mathrm{EM}}$, gray squares). Straight lines with slope equal to 1 are included manually to guide the eye. Intercepts correspond to the values of $K_{1}$ and $K_{n}$ and the slopes of a linear fit around $p_{50}\left(n_{50}\right.$, fit not shown for clarity) are summarized in Table 1. (A): pH 6.5, no L-lactate; (B) pH 6.5, $40 \mathrm{mM}$ L-lactate, the dashed line is the upper line of panel A; (C): pH 7.5, no L-lactate; (D) $\mathrm{pH} 7.8$, no L-lactate; (E) pH 7.8, $40 \mathrm{mM}$ L-lactate. Buffer: Tris/ $\mathrm{HCl} 50 \mathrm{mM}, \mathrm{CaCl}_{2} 20 \mathrm{mM}, 25 \%$ glycerol (v/v) at the desired pH and presence/ absence lactate.

a decrease in $p_{50}$ upon increasing the $\mathrm{pH}$ (Table 1$)$. Also, we find a pronounced L-lactate effect $\left(\Delta \log \left(p_{50} /\right.\right.$ Torr $) / \Delta \log ([$ L-lactate $] /$ $\mathrm{mM})=-0.31$ and -0.39 at $\mathrm{pH} 6.5$ and $\mathrm{pH} 7.8$ in solution, respectively). Cooperativity as quantified by the Hill coefficient at half-saturation is slightly affected by $\mathrm{pH}$ and L-lactate, but no unique trend could be identified (Table 1).

In an ideal scenario, the oxygen binding behavior of trapped states $\left(\mathrm{Oxy}^{\mathrm{EM}}\right.$ and Deoxy $\left.{ }^{\mathrm{EM}}\right)$ reflects the properties of the conformation(s) existing under the embedding conditions in solution. Then, the binding curves should exhibit either no cooperativity (if only one conformation is trapped) or negative cooperativity, if two or more conformers are present in a fixed relative amount. The latter situation is likely to arise in case of C. aestuarii hemocyanin, since it has been shown that four conformations are required to describe the cooperative and allosteric behavior of this hemocyanin, ${ }^{24}$ thus a mixture of two conformations at fully oxygenated or fully deoxygenated conditions is likely to occur.
Under all five conditions $\left(\mathrm{pH}, \pm_{\mathrm{L}}\right.$-lactate), encapsulation under deoxygenated conditions led to increased values for $p_{50}$, whereas encapsulation under oxygenated conditions led to a decrease in $p_{50}$. Furthermore, the Hill-coefficient decreased upon encapsulation. Thus, low-affinity and high-affinity conformations were selectively stabilized. One would further expect that common slopes for solution and Deoxy ${ }^{\mathrm{EM}}$ at 0 Torr exist, and also for solution and $\mathrm{Oxy}^{\mathrm{EM}}$ at 760 Torr. To check this, lines with slope $=1$ were added to the Hill-plots and by this a first estimation of the corresponding affinities was obtained (Fig. 1 and Table 1).

The simplest possible scenario was observed for the oxygen binding curves of the hemocyanin trapped at $\mathrm{pH}$ 6.5. Both Deoxy $^{\mathrm{EM}}$ and $\mathrm{Oxy}^{\mathrm{EM}}$ display non-cooperative oxygen binding behavior with $n_{50}$ values close to 1 . Common slopes for deoxygenated protein in solution and Deoxy ${ }^{\mathrm{EM}}$ seem to exist, and also for the oxygenated form in solution and Oxy ${ }^{\mathrm{EM}}$ (Fig. 1A and Table 1). These results suggest that the entrapment of 
Table 1 Oxygen binding properties of Carcinus aestuarii hemocyanin in solution and embedded into sol-gel matrix, as estimated from the Hill plots of Fig. 1A-E and 2. Oxy ${ }^{\mathrm{EM}}$, Deoxy ${ }^{\mathrm{EM}}$ identify hemocyanin embedded in the sol-gel in the oxygenated and deoxygenated state, respectively, before the oxygen binding experiments. $p_{50}, n$ and $K_{1}, K_{n}$ indicate the oxygen partial pressure at half-saturation, the cooperativity index and the affinity constant for oxygen at very low and very high saturation. Buffer: $50 \mathrm{mM} \mathrm{Tris} / \mathrm{HCl}, \mathrm{CaCl}_{2} 20 \mathrm{mM}, 25 \% \mathrm{glycerol}(\mathrm{v} / \mathrm{v})$, pH and lactate as indicated. The squares highlight pairs of values which are expected to be similar. Standard errors were estimated in cases, where 3 binding curves were available

\begin{tabular}{|c|c|c|c|c|c|c|}
\hline \multicolumn{6}{|c|}{ Lactate $0 \mathrm{mM}$} & \multirow{2}{*}{$\begin{array}{c}\# \\
\text { exp }\end{array}$} \\
\hline & & $P_{50}$ (Torr) & $\mathrm{n}$ & $\log K 1$ & $\log K n$ & \\
\hline \multirow{3}{*}{$\begin{array}{l}\mathrm{pH} \\
6.5\end{array}$} & $O x y^{E M}$ & $34 \pm 4$ & $0.89 \pm 0.04$ & -1.4 & -1.4 & 3 \\
\hline & Solution & $73 \pm 2$ & $2.8 \pm 0.3$ & -2.6 & -1.4 & 3 \\
\hline & $D_{e o x y}^{E M}$ & 220.8 & 0.9 & -2.6 & -2.6 & 2 \\
\hline & & & & & & \\
\hline \multirow{3}{*}{$\begin{array}{l}\text { pH } \\
7.5\end{array}$} & $O x y^{E M}$ & 12.1 & 1.1 & -1 & -1 & 2 \\
\hline & Solution & 23.1 & 3.3 & -2.2 & -0.5 & 2 \\
\hline & $\operatorname{Deoxy}^{E M}$ & 34.7 & 1.4 & -2.2 & -1 & 2 \\
\hline \multirow{3}{*}{$\begin{array}{l}\text { pH } \\
7.8\end{array}$} & $O x y^{E M}$ & $11 \pm 2$ & $1.36 \pm 0.06$ & -1.2 & -0.5 & 3 \\
\hline & Solution & $13 \pm 1$ & $2.3 \pm 0.4$ & 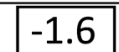 & -0.5 & 3 \\
\hline & Deoxy ${ }^{E M}$ & $22 \pm 2$ & $1.28 \pm 0.02$ & -1.6 & -1.2 & 3 \\
\hline \multicolumn{7}{|c|}{ Lactate $\mathbf{4 0} \mathrm{mM}$} \\
\hline & & $P_{50}$ (Torr) & $\mathrm{n}$ & & & \\
\hline \multirow{3}{*}{$\begin{array}{l}\mathrm{pH} \\
6.5\end{array}$} & $O x y^{E M}$ & $11 \pm 4$ & $0.89 \pm 0.06$ & -1 & -1 & 3 \\
\hline & Solution & $23 \pm 2$ & $1.35 \pm 0.09$ & -1.55 & -1 & 3 \\
\hline & $D e o x y^{E M}$ & $23 \pm 6$ & $0.8 \pm 0.1$ & -1.25 & -1.25 & 3 \\
\hline \multirow{3}{*}{$\begin{array}{l}\text { pH } \\
7.8\end{array}$} & $O x y^{E M}$ & 1.8 & 0.6 & -0.2 & -0.2 & 2 \\
\hline & Solution & $3.1 \pm 0.2$ & $2.8 \pm 0.7$ & -1.1 & -0.2 & 3 \\
\hline & $D e o x y{ }^{E M}$ & 8.3 & 0.8 & -1.1 & -1.1 & 1 \\
\hline
\end{tabular}

hemocyanin in two different conformational states (Oxy ${ }^{\mathrm{EM}}$ and Deoxy ${ }^{\mathrm{EM}}$ ) yields mainly the corresponding high- and low-oxygen affinity conformers. The low affinity conformer, which is represented by Deoxy ${ }^{\mathrm{EM}}$, cannot be fully saturated even at 760 Torr. In this case, the absorbance at saturation was estimated by fitting the available experimental data with a hyperbolic function. Also in presence of L-lactate, the conformation of the form entrapped in the oxy-form $\left(\mathrm{Oxy}{ }^{\mathrm{EM}}\right)$ seems to correspond to the conformer found in solution under oxygenated conditions (Fig. 1B and Table 1). The deoxy form (Deoxy ${ }^{\mathrm{EM}}$ ) exhibits an increased affinity in absence of oxygen compared to the deoxyform in solution. The Hill-coefficients might indicate a slight heterogeneity in the entrapped conformations $\left(n_{50}<1\right)$.

Hemocyanin entrapped at $\mathrm{pH} 7.5$ (Fig. 1C and Table 1) shows non-cooperative oxygen binding in $\mathrm{Oxy}^{\mathrm{EM}}$, but has still considerable residual cooperativity in Deoxy ${ }^{\mathrm{EM}}$. The asymptotes of the Hill-plots indicate similar affinities in the oxygenated state for the two entrapped conditions, which are lower than the one in solution. The affinities in the deoxygenated state fit into the expected pattern, with similar asymptotes for solution and $\mathrm{Deoxy}^{\mathrm{EM}}$, and a higher affinity for Oxy ${ }^{\mathrm{EM}}$.

Finally, at pH 7.8 under both entrapping conditions cooperativity is not completely hindered. However, the affinity of hemocyanin in solution and Deoxy ${ }^{\mathrm{EM}}$ at low oxygen concentration is similar and lower than the one of $\mathrm{Oxy}^{\mathrm{EM}}$. At high oxygen concentrations again hemocyanin in solution data seem to have the highest affinity, followed by $\mathrm{Oxy}^{\mathrm{EM}}$ and then Deox$\mathrm{y}^{\mathrm{EM}}$. Embedding hemocyanin in the presence of $40 \mathrm{mM}$ L-lactate increases the trapping effect of the sol-gel at pH 7.8 (Fig. 1E and Table 1). The Hill-coefficients indicate a mixture of conformations. However, the values for the Hill-coefficients are in this case only rough estimates, since they rely on data points covering only the upper part of the saturation curve.

\section{Model based analysis of oxygen binding data}

Taken together, the Hill-plots indicate that the highest affinity achievable for the investigated hemocyanin is about $K_{\mathrm{R}}=0.6$ $\operatorname{Torr}^{-1}$ (intercept $=-0.2$ ), whereas the lowest one is about $K_{\mathrm{T}}=$ 
0.002 Torr $^{-1}$ (intercept $=-2.2$ ). The intercepts between these limiting values could be either distinct conformations or a superposition of the $\mathrm{R}$ - and the T-state.

To determine the minimal number of conformations necessary to describe the functional data in solution and embedded in the sol-gel, data were analyzed based on different cooperative models in two steps. Due to the large number of parameters involved, a global fit of all data was not possible. Thus, first only the solution data were analyzed in a global fit based on the 3state, the 4-state and the Nested-MWC-model. Based on the ratio between the squared residuals and the degree of freedom, the three-state model would be sufficient (Table $\mathrm{S} 4 \dagger$ ). However, if for each condition ( $\mathrm{pH} 6.5$ with or without $40 \mathrm{mM}$ L-lactate, $\mathrm{pH} 7.5$, $\mathrm{pH} 7.8$ with or without $40 \mathrm{mM}$-lactate) the three data sets (solution, Deoxy ${ }^{\mathrm{EM}}$, Oxy ${ }^{\mathrm{EM}}$ ) were analyzed simultaneously based on a 2, 3 or 4-state model, it turned out that a total of four conformations is needed to describe all data (Table S5 $\dagger$ ).

Thus, analysis was repeated based on the Nested-MWCmodel, with part of the binding constants set constant to the value derived from solution data. This was necessary in those cases where clearly some conformations do not contribute to the binding curve. This procedure yielded the final, common set of binding constants, with the following values: $K_{\mathrm{tT}}=0.003$ Torr $^{-1}, K_{\mathrm{tR}}=0.02-0.03$ Torr $^{-1}, K_{\mathrm{rR}}=0.1-0.2$ Torr $^{-1}, K_{\mathrm{rT}}=0.9 \pm$ 0.1 Torr $^{-1}$ (see Table S6 and ESI† for details).

To get information about the conformational distribution, each binding curve was then analyzed individually with the binding constants set constant to the values given in Table S6. $\dagger$ For binding curves with positive cooperativity $\left(n_{50}>1\right)$, the allosteric equilibrium constants for each condition were fitted to the data (Table S7†). For cases with none or negative cooperativity the fraction of molecules in each conformation was fitted to the data based on a sum of hyperbolic functions (eqn (6) and Table S8 $\dagger$ ). For comparison of these fixed conformational distributions with the initial and final states in solution, the fraction of molecules in each conformation was calculated for the latter for 0 Torr and 760 Torr (Table S8†). For pH $6.5 \pm$ L-lactate and pH $7.8+$ L-lactate the agreement is reasonable. For $\mathrm{pH} 7.5$ and 7.8, where the embedded protein still undergoes conformational transition, the calculated distributions agree in absence of oxygen, and seem to differ in presence of oxygen.

Overall, the pattern found in the calculated conformational distributions mirrors the one found by comparing the slopes in the Hill-plot, as expected, but revealed that four conformations exists.

\section{Structural characterization of hemocyanin conformers: spectroscopic studies}

Spectroscopic analysis was carried out on the high-affinity $\left(\mathrm{Oxy}^{\mathrm{EM}}\right)$ and low-affinity (Deoxy ${ }^{\mathrm{EM}}$ ) conformers and compared with the protein in solution to define the structural features that govern the functional properties of the different conformers. The choice was made to work at $\mathrm{pH} 7.8$, a value close to the physiological one.

In Fig. 2 the near-UV/visible CD spectra of hemocyanin in the presence of oxygen (2A) and in absence of oxygen (2B) are
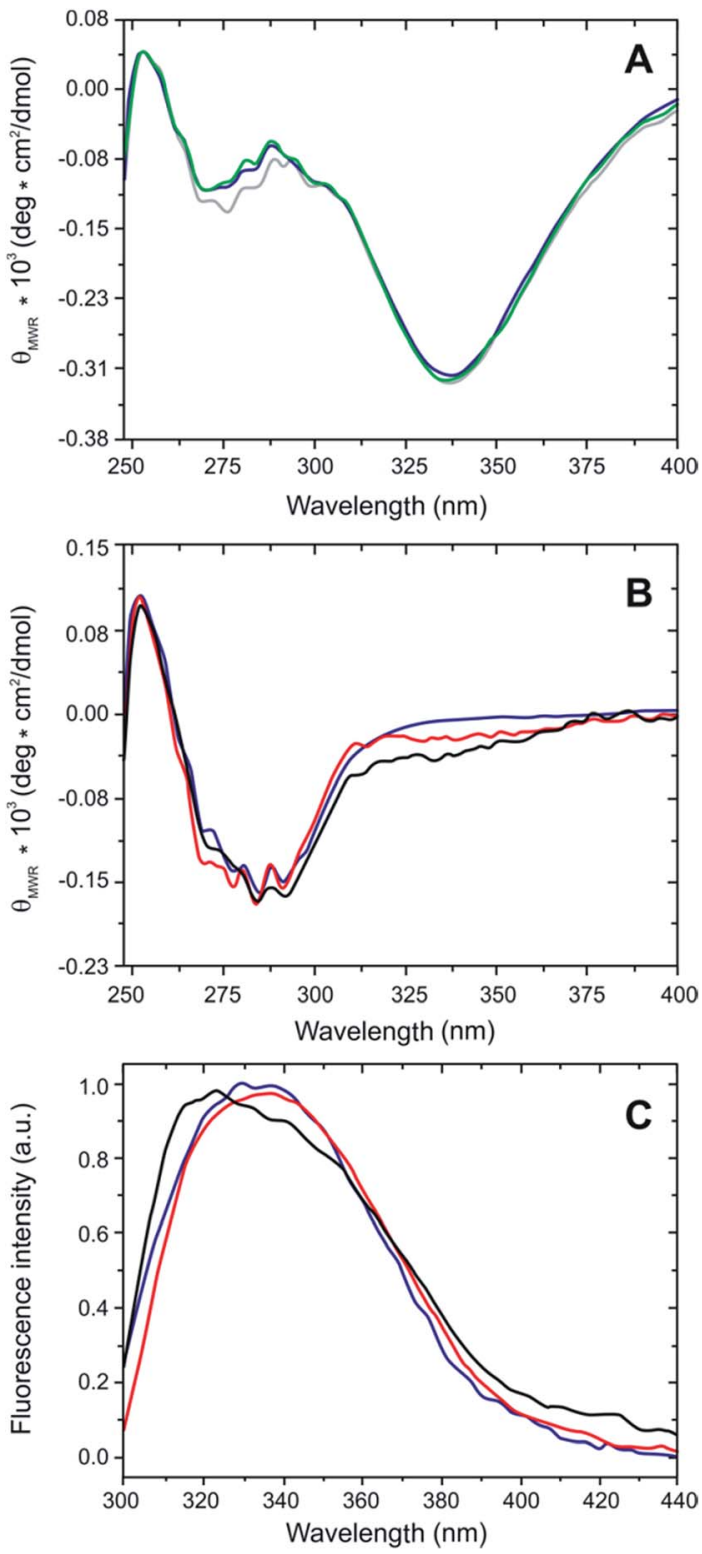

Fig. 2 Spectroscopic properties of different hemocyanin forms in 50 $\mathrm{mM}$ Tris/ $\mathrm{HCl}, \mathrm{CaCl}_{2} 20$ mM, 25\% glycerol (v/v) pH 7.8. (A) Near-UV/ visible $C D$ spectra of oxygenated hemocyanin in solution (blue), Oxy760 (green), Deoxy760 (gray). (B) Near-UV/visible CD spectra of deoxygenated hemocyanin in solution (blue), Oxyo (black) Deoxy ${ }_{0}^{\mathrm{EM}}$ (red) at $\mathrm{pH}$ 7.8. (C) Fluorescence emission spectra (excitation wavelength at $278 \mathrm{~nm}$ ) of deoxygenated hemocyanin in solution (blue), Oxyo ${ }_{0}^{E M}$ (black) or as Deoxyo (red) at pH 7.8. Spectra are normalized to the maximum emission intensity.

presented. Oxygenated hemocyanin $\mathrm{CD}$ is dominated by a band with negative ellipticity centered at $338 \mathrm{~nm}$ that corresponds to the peroxide-to- $\mathrm{Cu}$ (II) ligand-to-metal charge-transfer transition and is very similar for Deoxy ${ }_{760}^{\mathrm{EM}}, \mathrm{Oxy}_{760}^{\mathrm{EM}}$ and the protein in solution, ${ }^{37}$ indicating that the ellipticity of the peroxide complex of oxygenated hemocyanin is the same for both the protein in solution and in sol-gel. In contrast, the CD spectral shape in the aromatic region reveals differences between the protein embedded in sol-gel and in solution. In particular, although the 
spectra of $\mathrm{Oxy}_{760}^{\mathrm{EM}}$ and oxy-hemocyanin in solution are almost indistinguishable, Deoxy ${ }_{760}^{\mathrm{EM}}$ shows significantly higher negative ellipticity in the $260-280 \mathrm{~nm}$ range where tyrosine residues contribute to the spectrum. ${ }^{37}$ The deoxygenated hemocyanin CD spectra (Fig. 2B) lack, as expected, the negative band at $345 \mathrm{~nm}$ due to the removal of peroxide, yet the residual negative ellipticity at this wavelength indicates that the sample still contains $\sim 10 \%$ of oxygenated hemocyanin under the conditions of CD measurements. Thus, no safe conclusion can be drawn from the spectral features in the near-UV due to the interference of residual the $345 \mathrm{~nm}$ band that has a variable different intensity in the various preparations.

Fluorescence emission spectra of deoxygenated hemocyanin in solution or gel-entrapped are presented in Fig. 2C. The corresponding spectra of oxygenated hemocyanin were not compared since the fluorescence emission of this form is strongly quenched by resonance energy transfer to the copper peroxide complex in the active site. ${ }^{38}$ Upon excitation at $278 \mathrm{~nm}$, where both tyrosine and tryptophan residues can be excited, the shape of the spectra shows some distinct differences. Although deoxygenated hemocyanin in solution and Deoxy ${ }_{0}^{\mathrm{EM}}$ have a maximum emission near $340 \mathrm{~nm}$, in case of $\mathrm{Oxy}_{0}^{\mathrm{EM}}$ a shift of the maximum peak of emission to $320 \mathrm{~nm}$ is observed. This shift in the maximum emission can be attributed to different contribution of Tyr and Trp residues to the overall fluorescence emitted by the protein in the two cases. ${ }^{39}$ Accordingly, the emission spectra obtained upon excitation at $294 \mathrm{~nm}$ (where

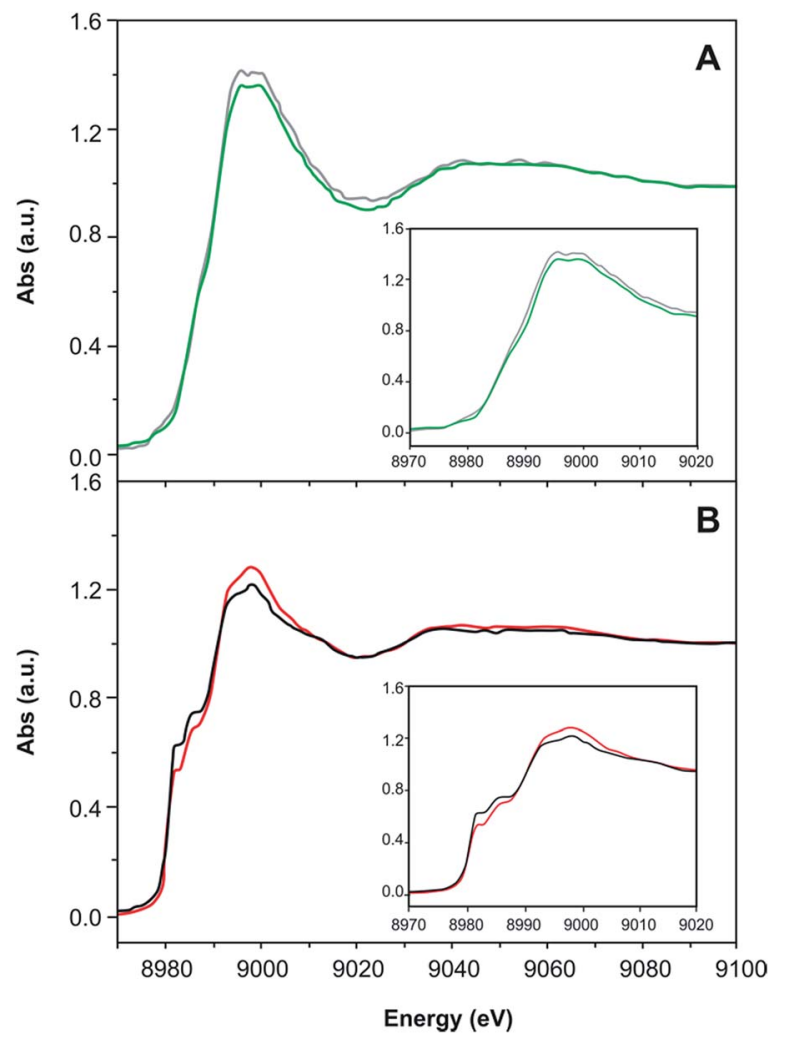

Fig. 3 X-ray absorption spectra of Deoxy760 (A, gray), Oxy760 (A, green), $\operatorname{Deoxy}_{0}^{\mathrm{EM}}\left(\mathrm{B}\right.$, red), and $\mathrm{Oxy}_{0}^{\mathrm{EM}}(\mathrm{B}$, black) at $\mathrm{pH} 7.8$. tryptophan residues contribute almost exclusively) do not show any difference in the various hemocyanin forms.

To determine if the changes of the oxygen affinity are due to different coordination geometries of the active site, X-ray absorption spectra for hemocyanin embedded in sol-gel were collected in presence and absence of oxygen. Coordination geometry of copper in the active site of hemocyanin in solution was already evaluated by XAS, ${ }^{22}$ showing distinct signatures in the X-ray absorption spectra of the oxygenated and the deoxygenated form. XAS measurements of hemocyanin embedded into the sol-gel matrix display the typical line shape described for hemocyanin in solution. In particular the difference spectra between deoxy- and oxy-hemocyanin evidence two main absorbance peaks at $\sim 8982 \mathrm{eV}$ and $\sim 8985 \mathrm{eV}$, which have been assigned to the $1 \mathrm{~s} \rightarrow 4 \mathrm{p}_{\mathrm{z}}$ and $1 \mathrm{~s} \rightarrow(\mathrm{s}+\mathrm{p})^{*}$ transitions, respectively. ${ }^{20,21}$ The spectra of $\mathrm{Oxy}^{\mathrm{EM}}$ or Deoxy ${ }^{\mathrm{EM}}$ recorded in presence of oxygen do not show significant differences in the XANES region (Fig. 3A). In contrast, in the deoxygenated state the spectra of the Deoxy ${ }^{\mathrm{EM}}$ and $\mathrm{Oxy}^{\mathrm{EM}}$ conformers (Fig. 3B) are characterized by different intensities of the transitions $1 \mathrm{~s} \rightarrow 4 \mathrm{p}_{\mathrm{z}}$ and $1 \mathrm{~s} \rightarrow(\mathrm{s}+\mathrm{p})^{*}$, which can be correlated to the displacement of $\mathrm{Cu}(\mathrm{I})$ from the plane defined by the three histidines. The lower intensity of the $8982 \mathrm{eV}$ band of Deoxy ${ }_{0}^{\mathrm{EM}}$ suggests a greater degree of distortion toward pseudotetrahedral geometry than the active site of $\mathrm{Oxy}_{0}^{\mathrm{EM}}$.

\section{Structural characterization of hemocyanin conformers: small angle scattering studies}

Details on SAXS data on sol-gel samples in the absence of proteins and under different conditions $(\mathrm{pH}$, glycerol content and aging time) are given in ESI together with the best fitting parameters obtained with the Teixeira model (Table S1 $\dagger$ ). Among the fitting parameters, the most relevant one is the correlation distance $\xi$, that accounts for the average pores' dimension. According to this parameter, under the conditions employed for measuring the oxygen affinities of the different states of hemocyanin in the sol-gel $(\mathrm{pH}=7.8,25 \%$ glycerol and 2 days of aging time) the pore size in the case of empty sol-gel is $\approx 328 \AA$.

SANS measurements were carried out at $\mathrm{pH} 7.8$ to characterize the structure of the $\mathrm{Oxy}^{\mathrm{EM}}$ and Deoxy ${ }^{\mathrm{EM}}$ conformers, both in the oxygenated and deoxygenated state, under the same conditions used to derive their oxygen affinities. SANS is particularly suitable to this aim because, using the contrast variation technique, the sol-gel can be made invisible by adjusting the content of $\mathrm{D}_{2} \mathrm{O}$ in the solution. ${ }^{40}$ SANS data of the sol-gel matrices without hemocyanin recorded as a function of the deuteration degree $x_{\mathrm{D}}$ reveal a matching point between bulk solvent and sol-gel at $x_{\mathrm{D}}=0.720 \pm 0.001$, a value quite similar to the one calculated on the basis of the chemical composition of the matrix (see ESI $\uparrow$ for further details). Hence, SANS data of hemocyanins embedded in the sol-gel at $x_{\mathrm{D}}=0.720$ were recorded and analyzed to unravel the quaternary structure of the $C$. aestuarii hemocyanin in the sol-gel.

As described in the experimental section, the SANS curves from embedded conformers in oxy- and deoxygenated conditions in absence and presence of $40 \mathrm{mM}$ L-lactate were analyzed 


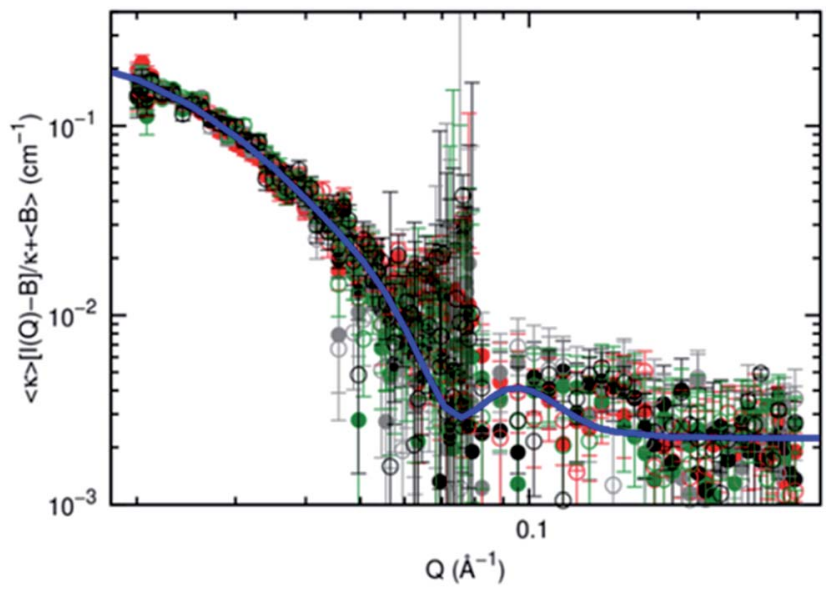

Fig. 4 Log-log SANS curves of different hemocyanin states in buffer $\mathrm{pH} 7.8$ and $x_{\mathrm{D}}=0.720$ normalized to the average scaling factor $\langle\kappa\rangle$ and the average background $\langle B\rangle$. Filled and open circles refer to the protein in the absence and in the presence of $40 \mathrm{mM}$ L-lactate, respectively. Colors refer to: Deoxy ${ }_{0}^{\mathrm{EM}}$ (red); Deoxy Oxy 760 (green). Solid blue line is the best fit obtained with QUAFIT.

by means of the new QUAFIT method. To take into account the effect of the experimental uncertainty of the scattering curves (mainly due to the incoherent scattering that affects the signal at high $Q$ ) on the fitting parameters, the analysis was repeated several times, by moving the experimental points within their error bars. The results show that among the eight samples obtained under different conditions, the differences in the values of the three Euler angles $\alpha, \beta$ and $\gamma$, which define the mutual position of the two hexamers, and in the hexamers' distance $d$ do not statistically differ from the differences within the repeated analysis of a single sample. The unique parameter that distinguishes the samples is the scaling factor $\kappa$, which can be considered a correction factor of the nominal protein concentration $c$ (see eqn (7)). The difference in the true protein concentration can be attributed to possible variations in the protein embedding and rinsing processes during sample preparation. Consequently, SANS data normalized at the same average values $\langle\kappa\rangle$ and $\langle B\rangle$, reported in Fig. 4 in logarithmic
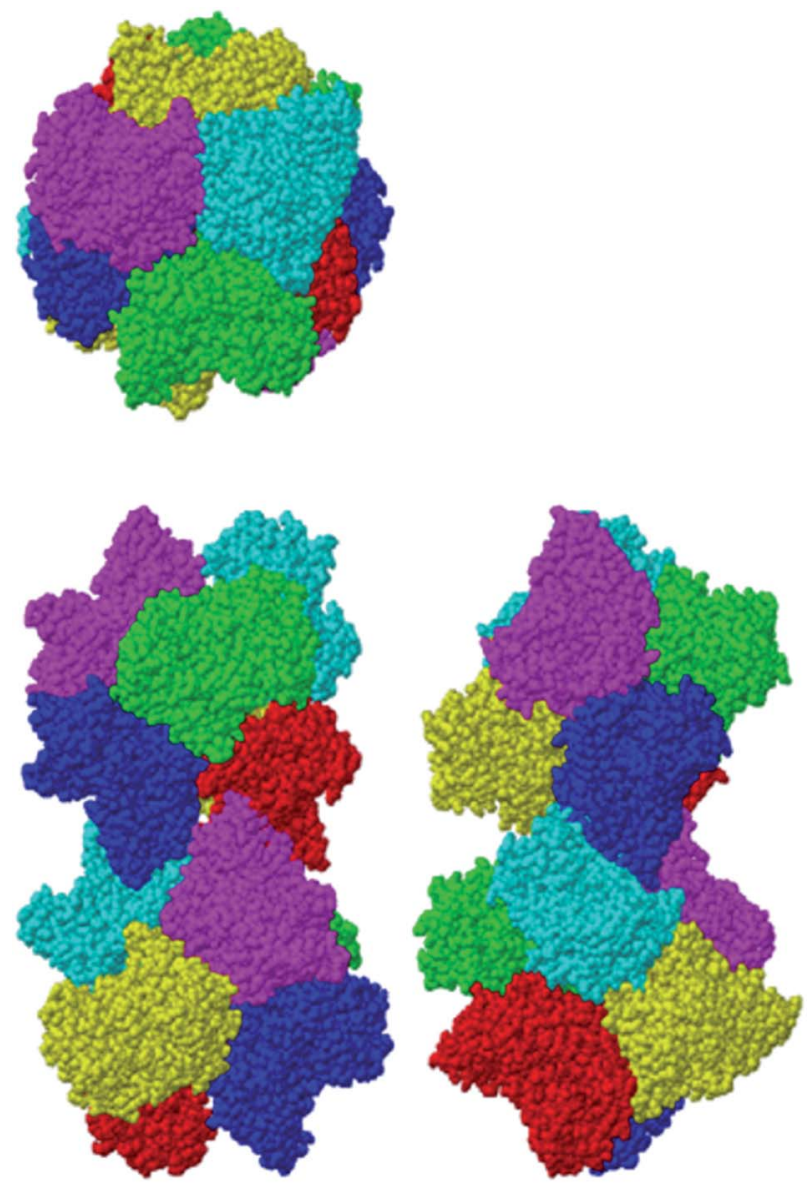

Fig. 5 Structure of the hemocyanin hexamer (upper) and dodecamer (lower) obtained by fitting the ensemble of the SANS curves with the QUAFIT software. The three views are drawn with RASMOL by using the spacefill option.

scale, appear to be comparable within their experimental uncertainties. The solid line in the figure refers to the best fit obtained by QUAFIT by considering a unique form factor for all the experimental SANS curves. Fitting parameters are reported in Table 2, whereas the structure of the unique dodecamer is shown in Fig. 5.

Table 2 QUAFIT fitting parameters. Top frame refer to the single curve parameters: the scaling factor $\kappa$ and the flat background $B$. Bottom frame report the common parameters: the three Euler angles $\alpha, \beta$ and $\gamma$ that define the relative orientation of the two hexamers within the dodecamer; the distance $d$ between the centers of the two hexamers; the relative mass density $d_{w}$ of the hydration water

\begin{tabular}{|c|c|c|c|c|}
\hline Lactate (mM) & 0 & 40 & 0 & 40 \\
\hline Deoxy $_{760}^{E M}$ & $0.35 \pm 0.03$ & $0.33 \pm 0.03$ & $0.0022 \pm 0.0002$ & $0.0021 \pm 0.0002$ \\
\hline $\mathrm{Oxy}_{0}^{\mathrm{EM}}$ & $0.29 \pm 0.03$ & $0.34 \pm 0.03$ & $0.0022 \pm 0.0002$ & $0.0022 \pm 0.0002$ \\
\hline $\mathrm{Oxy}_{760}^{\mathrm{EM}}$ & $0.36 \pm 0.04$ & $0.20 \pm 0.02$ & $0.0021 \pm 0.0002$ & $0.0023 \pm 0.0002$ \\
\hline $120 \pm 90$ & $60 \pm 10$ & $200 \pm 100$ & $100 \pm 20$ & $1.03 \pm 0.02$ \\
\hline
\end{tabular}


These results demonstrate that the protein in the sol-gel maintains its aggregation state and that the different oxygen binding properties induced by allosteric effects do derive from the presence of different conformers.

\section{Discussion}

Most biological processes, like metabolism, catalysis, signal transduction and gene regulation are governed by cooperative and allosteric mechanisms. ${ }^{\mathbf{4 1}}$ In oxygen transport proteins these mechanisms lead to adjustment of the oxygen affinity to environmental and physiological oxygen concentration and additionally regulation by binding of heterotropic allosteric effectors. This type of regulation requires the presence of at least two different conformations. The description of cooperative oxygen binding may involve even more than two conformational states, which are typically induced by interactions between subunits at the tertiary and quaternary structure level. ${ }^{\mathbf{4 2 , 4 3}}$ Due to the scarce crystallographic information available, our understanding of the molecular basis of cooperative interactions in the case of the high molecular weight oxygen carriers like hemocyanins is still very limited.

In this scenario, we focused on hemocyanin of the crustacean $C$. aestuarii, in which the existence of strongly hierarchical levels of interactions, within and between hexameric allosteric units, has been reported. ${ }^{24}$ Entrapment in a sol-gel matrix was used here to stabilize individual protein conformations, with the aim to define the structural features of the different conformations and their relation to their particular oxygen affinity.

The oxygen binding process of hemocyanins has been generally described by an extension of the classical MWC model that considers the existence of two levels of hierarchical allosteric interactions between $\mathrm{R}$ and $\mathrm{T}$ states for high molecular weight oligomers (dodecamers or more) as well as between $\mathrm{r}$ and $t$ states within the hexameric building blocks. ${ }^{11,12}$ For $C$. aestuarii hemocyanin four different hierarchical conformations were previously identified ${ }^{24}$ and this finding is supported by the present study. The agreement between the two sets of data is reasonably good in three cases $K_{\mathrm{tT}}=0.003$ and 0.006 Torr $^{-1}, K_{\mathrm{tR}}$ $=(0.02-0.036)$ and 0.016 Torr $^{-1}, K_{\mathrm{rT}}=0.88 \mathrm{Torr}^{-1}$ and 1.00 Torr $^{-1}$. Only in case of the medium affinity the difference is larger $\left(K_{\mathrm{rR}}=0.081-0.18\right.$ in the present study, and 0.7 in the previous one). Also the $p_{50}$-values tend to be lower in the present study than in the earlier one and the extent of the Bohr-effect is somewhat reduced $\left(\Delta \log p_{50} / \Delta \mathrm{pH}-0.55\right.$ and -0.68$)$. Overall, the discrepancy is within the variability expected for this kind of biological samples.

Hemocyanin from $C$. aesturarii displays a significant L-lactate effect $\left(\Delta \log \left(p_{50} /\right.\right.$ Torr $\left.)\right) / \Delta \log ([\mathrm{L}$-lactate $] / \mathrm{mM})=-0.31$ and -0.39 at $\mathrm{pH} 6.5$ and $\mathrm{pH}$ 7.8, respectively. The range observed in crustacean hemocyanin is between zero and $-0.56 .{ }^{44}$ Analysis of the oxygen binding curves in absence and presence of L-lactate allowed determining the changes in conformational distribution at different $\mathrm{pH}$ due to addition of L-lactate. The interaction of L-lactate with this hemocyanin in the oxygenated state was investigated directly based on isothermal titration calorimetry
(ITC) before. ${ }^{22}$ The ITC data indicated that binding characteristics are different at $\mathrm{pH} 6.5$ and $\mathrm{pH}$ 8.3. This observation is not surprising in light of our data: at $\mathrm{pH} 6.5$ addition of L-lactate leads to a conformational transition from $\mathrm{tR}$ to $\mathrm{rR}$. Thus, the reaction heats measured during titration of L-lactate are partially caused by conformational transitions. In contrast, at pH 7.8 (and even more so at pH 8.3 due to the Bohr-effect) under oxygenated conditions hemocyanin is present mainly in conformation rT, and addition of L-lactate shifts the distribution further into this direction. It has been shown before that concomitantly occurring conformational changes can alter the apparent stoichiometry of binding in an ITC experiment, ${ }^{\mathbf{4 5}}$ which could be the reason for the better fit of a model with two different binding sites in case of $\mathrm{pH}$ 6.5. ${ }^{22}$ Based on the same considerations the results of flash-photolysis experiments at $\mathrm{pH}$ 6.5 and $\mathrm{pH} 7.8^{22}$ can be rationalized: at $\mathrm{pH} 6.5$ the rate of oxygen-rebinding increases from $70 \mathrm{~s}^{-1}$ to about $82 \mathrm{~s}^{-1}$ upon addition of L-lactate, reflecting a transition from mainly tR to $\mathrm{rR}$. In contrast, at $\mathrm{pH} 7.8$ only a minor increase from $82 \mathrm{~s}^{-1}$ to $85 \mathrm{~s}^{-1}$ was observed.

Entrapment of hemocyanin in sol-gel altered the conformational distributions but not the quaternary structure, namely the molecular dimensions and spatial arrangement as shown by SANS. We could also show that the encapsulation of hemocyanin preserves the active site structure of the protein by XAS. Furthermore, XAS spectra in absence and presence of oxygen show similar differences as reported before for hemocyanin in solution. ${ }^{22}$

The pores of empty sol-gel (at pH 7.8, 25\% glycerol and 2 days aging the diameter of pores $\approx 328 \AA$, Table $\mathrm{S} 1 \dagger$ ) have dimensions comparable with the size of hemocyanin dodecamer (maximum dimension about $225 \AA^{25}$ ), suggesting that the shape of the porous cavities are suited to entrap a single protein molecule. This dimension, however, changes as a function of $\mathrm{pH}$ and sol-gel aging time. As reported in Table $\mathrm{S} 1, \dagger$ the dimensions at $\mathrm{pH} 7.5$ and $6.5(\approx 70$ and $98 \AA)$ are smaller than the size of hemocyanin, suggesting that during the gelling process in the presence of hemocyanin the pore cavities are influenced by the presence of the protein, a case similar to that described by Luo et al. $^{\mathbf{4 0}}$ for green fluorescent protein. Therefore, the presence of large cavities that may contain more than one protein molecule and/or large amounts solvent molecules is unlikely. On these premises, it can be expected that the sol-gel is capable to block the conformational transitions of the hemocyanin induced by changing the oxygen concentration, and by this enable to characterize particular conformers, both under oxygenated and deoxygenated conditions. With these premises, the characterization of embedded hemocyanin was carried out on samples prepared with a standard protocol, as outlined above and in the experimental section, to avoid artifacts due to time-dependent changes of the sol-gel structure. Furthermore, the timespan of spectroscopic and SANS measurements is negligible when compared with the time scale of such changes of the sol-gel.

Overall, the oxygen binding properties of embedded hemocyanin are compatible with the selection of distinct conformations characteristic for aerobic or anaerobic conditions. In all 
cases, the $p_{50}$ of $\mathrm{Oxy}{ }^{\mathrm{EM}}$ and Deoxy ${ }^{\mathrm{EM}}$ was, as expected, higher and lower than the value for the protein in solution. Data obtained at different $\mathrm{pH}$-values revealed that the ability of the matrix to inhibit the allosteric transitions depends on $\mathrm{pH}$ and also on the presence of L-lactate. Analysis of the binding data based on different cooperative models indicate that at $\mathrm{pH} 6.5$ in absence and presence of L-lactate and at $\mathrm{pH} 7.8$ in presence of $\mathrm{L}^{-}$ lactate, conformations are effectively blocked at the distribution present upon embedding. In these cases the binding curves seem to be dominated by only two conformations already present in solution (Fig. 6). For pH 7.5 and pH 7.8 significant conformational transitions occur. These are likely related to altered charges due to protonation of both allosteric and nonallosteric sites compared to lower $\mathrm{pH}$-values, which result in reduced interactions between protein and sol-gel thus reducing trapping efficiency. Additionally, at $\mathrm{pH} 7.8$ the free energy differences between the different conformations are smaller, thus making conformational transitions more likely. At low $\mathrm{pH}$ the protein is more positively charged offering more electrostatic interactions with the negatively charged inside surface of the matrix's pores. Electrostatic interactions between the embedded protein and the sol-gel matrix were described as pivotal for blocking a given protein conformation..$^{5,31}$ The role of net-charge with respect to the entrapment properties of a solgel matrix was also previously reported ${ }^{46}$ showing that

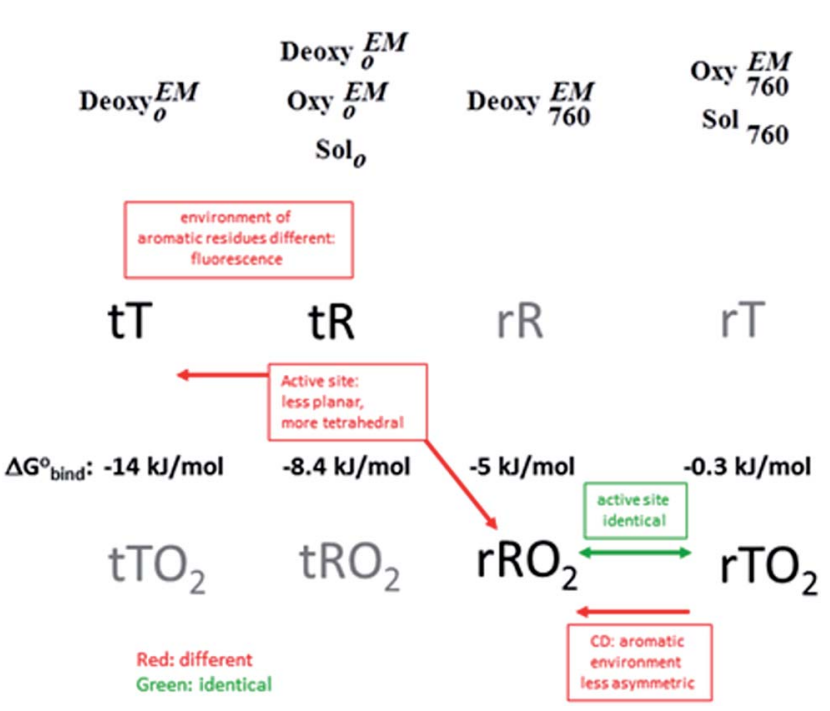

Fig. 6 Overview of the different conformations and their characteristics. The analysis of oxygen binding data revealed four different conformations ( $t T, t R, r R, r T){ }^{8}$ differing in their free energy for oxygen binding, which were dominantly populated under the experimental conditions indicated in the first row. Of the full scheme, only four conformations (deoxy tT and tR and oxy rR and $\mathrm{rT}$ ) could be stabilized and thus amenable for spectroscopic analysis (black shapes). Active site spectroscopy employing XAS indicate that the two deoxygenated states are less planar than the two oxygenated ones, with tR representing the most planar state. Conformational differences of the oxygenated states were revealed by $C D$-spectroscopy, indicating a less asymmetric environment of the aromatic residues in the conformation $r R$. The two deoxygenated state also seem to be different in the local environment of the tryptophans, as revealed by fluorescence spectroscopy. positively charged molecules are more strongly hindered in their motion. Thus, one would expect that screening of charges leads to less effective encapsulation. Indeed, addition of 500 $\mathrm{mM} \mathrm{NaCl}$ leads to less effective entrapment under oxygenated conditions: the cooperativity index $n_{50}$ and the $p_{50}$ increase $\left(\mathrm{Oxy}^{\mathrm{EM}}\right.$ in $500 \mathrm{mM} \mathrm{NaCl}$ at pH 6.5 exhibits $n_{50}=1.26$ and $p_{50}=$ 61.55 Torr versus $n_{50}=0.89$ and $p_{50}=33.84$ in absence of $\mathrm{NaCl}$ ), thus in presence of $\mathrm{NaCl}$ the oxygen binding properties of the entrapped hemocyanin are closer to the solution situation (in $500 \mathrm{mM} \mathrm{NaCl}: p_{50}=64.74, n_{50}=2.51$ ). Addition of $500 \mathrm{mM}$ $\mathrm{NaCl}$ leads to an opposite effect on the $p_{50}$-values of hemocyanin in solution (a decrease from 74.70 Torr to 64.74 Torr), showing that the effect described above is sol-gel specific. However, if mainly the overall charge of the protein is responsible for the entrapment efficiency it is difficult to explain that in presence of L-lactate at pH 7.8 efficient entrapment occurs, indicating an additional factor for efficient arrestment of conformational transitions. Possibly, if the protein can change rapidly between two conformations during the gelling-process (as at $\mathrm{pH} 7.5$ and pH 7.8 in absence of L-lactate), the pores fit less well to the protein than in a situation where most molecules are in the same conformation, and thus the transition probability for each molecule is much smaller (as at pH 7.8 in presence of $\mathrm{L}$-lactate), allowing a more adapted shape of the pore.

Several authors showed that the interactions of the matrix with embedded organic chromophores, such as tetrapyrrolic macrocycles, may affect their spectroscopic properties. ${ }^{47-52}$ In the case of hemocyanin, however, two points should be taken into consideration: (i) the active site is located in a hydrophobic cavity deeply buried in the protein; ${ }^{14-16}$ (ii) changes in oxygen affinity are dictated by changes in the copper-copper and copper-ligands distances of the copper-imidazole complex. ${ }^{17-23}$ Thus, the interactions of the protein with the pore walls are likely restricted to its surface without direct effect on the active site structure and the different binding constants determined by oxygen equilibria are attributable to the entrapping of distinct protein conformations.

Although at $\mathrm{pH} 7.8$ the conformations were not arrested completely, the effect was strong enough to allow detection of alterations in certain structural features (Fig. 6). Differences in active site geometry were evident between $\operatorname{Deox}_{0}^{\mathrm{EM}}$ and $\mathrm{Oxy}_{0}^{\mathrm{EM}}$ samples. Furthermore, fluorescence spectra of these states were different upon excitation at $280 \mathrm{~nm}$, although the spectrum of hemocyanin in solution in absence of oxygen is indistinguishable from Deoxy ${ }_{0}^{\mathrm{EM}}$. Under oxygenated conditions, the active site geometry is the same $\left(\operatorname{Deoxy}_{760}^{\mathrm{EM}}=\mathrm{Oxy}_{760}^{\mathrm{EM}}\right)$ but the CD-spectrum in the aromatic region differs. Here, solution data are very similar to the one for $\mathrm{Oxy}_{760}^{\mathrm{EM}}$.

Analysis of binding data at $\mathrm{pH} 7.8$ in frame of the NestedMWC-model yielded values for the corresponding allosteric equilibrium constants with considerable uncertainties in some cases, in particular in case of oxy-embedded hemocyanin. Thus, for comparison with the structural data we did not only calculate the corresponding conformational distributions for the best fit parameters (Table S8 $\dagger$ ), but we also addressed the stability of the conformations upon changes in the values of $l_{\mathrm{T}}$, $l_{\mathrm{R}}$ and $\Lambda$ within their error ranges. Among the calculated 
binding curves resulting from all possible combinations of parameters, only those reasonably close to the best fit situation, as indicated by a moderate increase in the sum of squared residuals $\mathrm{SS}^{2}\left(\mathrm{SS}^{2}<\mathrm{SS}_{\min }{ }^{2}\right.$, see $\mathrm{ESI} \dagger$ for details), were further considered. In case of solution data and Deoxy ${ }^{\mathrm{EM}}$ the conformational distribution under oxygenated conditions is robust. For deoxygenated conditions a significant variability in the relative amount of $\mathrm{tR}$ and $\mathrm{tT}$ is found, but conformation $\mathrm{tT}$ is always present to at least $40 \%$. In total, a common conformational distribution for solution data and Deoxy ${ }^{\mathrm{EM}}$ in absence of oxygen is supported. In case of $\mathrm{Oxy}^{\mathrm{EM}}$ the range of distributions being in agreement with $\mathrm{SS}^{2}<2 \mathrm{SS}_{\min }{ }^{2}$ is rather broad. We selected a region which complies with the prediction of the structural information as summarized in Fig. S9, $\uparrow$ identified the critical parameter $(\Lambda=-9.156)$ and re-analyzed the binding curve with this constraint. The resulting parameters delivered a fit with $\mathrm{SS}^{2}=1.5 \mathrm{SS}_{\min }{ }^{2}$ and $75 \% \mathrm{rT}$ at 760 Torr, $92 \%$ tR at 0 Torr, which is in nice agreement with the solution data. Thus, overall a consistent picture of the conformations under different conditions could be generated by combining all available data, as summarized in Fig. 6.

In this frame, the differences in the active site geometry as observed by XAS can now be attributed to particular conformations. Oxygenated conformation rT and rR have very similar spectra, although deoxygenated $\mathrm{tT}$ and $\mathrm{tR}$ differ, with deoxy-tR displaying a higher intensity at $8982 \mathrm{eV}$. The intensity of the band at this energy is correlated with the extent of displacement of the $\mathrm{Cu}(\mathrm{I})$ from the plane defined by the three ligand histidines. $^{20,21}$ A greater degree of distortion toward pseudotetrahedral geometry is reflected by a lower intensity, suggesting that deoxy-tR exhibits a more trigonal planar geometry at the active site. This would mean that a more trigonal geometry leads to higher affinity $\left(K_{\mathrm{tR}}=0.021 \pm 0.002 \mathrm{Torr}^{-1}\right.$, Table S6 $\left.\dagger\right)$ than a conformation with larger distortion as found for tT. To model the active site geometry of the different conformers, suitable information can be obtained by X-ray crystallography studies. Trigonal coordination geometry characteristic of deoxytR conformer (Fig. 7, left panel) can be represented by the X-ray
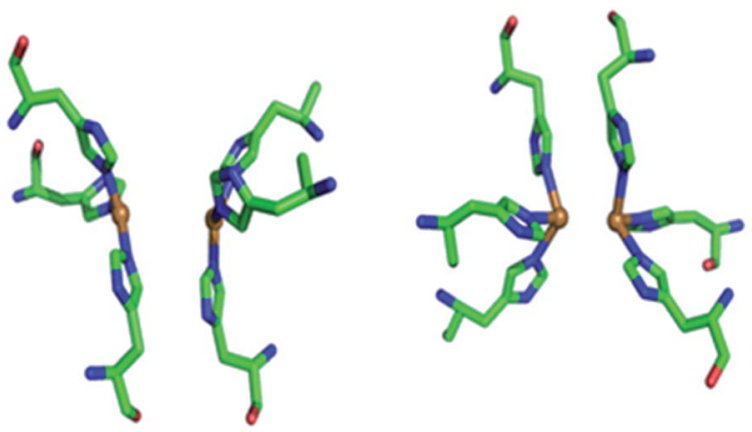

Fig. 7 Schematic representation of the dinuclear active site geometries of the two deoxygenated conformers of $\mathrm{Hc}$. These models have been generated starting from the two paradigmatic crystal structures available: left panel; trigonal coordination geometry of the deoxy-form of Limulus polyphaemus subunit II (pdb: 1LLA ${ }^{15,16}$ ). Right panel; pseudo-tetrahedral geometry of the deoxy-form of Panulirus interruptus (pdb: $1 \mathrm{HCY}^{14}$ ). crystal structure of the deoxy-form of Limulus polyphaemus subunit II. In this complex, the $\mathrm{N} \varepsilon$ imidazole nitrogens of three histidines are located at a distance of 1.9-2.2 $\AA$ from the copper and define a plane from which the copper is displaced by $0.3 \AA$ (pdb: 1LLA ${ }^{\mathbf{1 5 , 1 6}}$ ). Moreover, a strong similarity exits between the k-edge spectrum of $\mathrm{Cu}(\mathrm{I})$ in reduced $\mathrm{SOD}^{22}$ and that of the high affinity form of deoxy-hemocyanin in terms of the relative intensity of the $1 \mathrm{~s}-4 \mathrm{p}_{\mathrm{z}}$ band compared with the edge jump. The X-ray crystal structure of reduced SOD (pdb: 1dsw) shows a trigonal coordination geometry for $\mathrm{Cu}(\mathrm{I})$, with three nearly equal $\mathrm{Cu}-\mathrm{N}$ bond lengths and angles of $120^{\circ}$ between these bonds.

The pseudo-tetrahedral geometry of the deoxy-tT conformer active site can be supported by the X-ray crystal structure of Panulirus interruptus deoxyHc at $\mathrm{pH} 4.8$ (pdb: $1 \mathrm{HCY}) .{ }^{14}$ In this structure, two $\mathrm{N} \varepsilon$ atoms of imidazole ligands and one copper atom lie nearly in plane, with $\mathrm{Cu}-\mathrm{N}$ bond lengths of $1.9 \AA$. A third histidine $\mathrm{N} \varepsilon$ is located perpendicular to this plane at $2.7 \AA$ (Fig. 7 right panel).

SANS data unambiguously show a dodecameric organization of $C$. aestuarii hemocyanin. The SANS-data obtained under different conditions did not reveal significant differences, but yielded a unique dodecameric structure. The average interhexameric distance of $100 \AA$, calculated as distance between the centers of the two hexamers, well compares with the value of $106 \AA$ determined by SAXS on C. aestuarii hemocyanin in solution. ${ }^{25}$ However, due to the low resolution of SANS measurements this can not exclude slight differences in the orientation or difference of the two hexamers within the dodecamer. The differences in aromatic CD under oxygenated conditions indicate a different local environment of tyrosines if conformations rR and rT are compared. The fluorescence data indicate differences in the aromatic residues also for conformation $\mathrm{tR}$ and $\mathrm{tT}$. However, the details of the alterations in the environment of aromatic residues can only be clarified based on crystallographic structures, which are not yet available with sufficient resolution for hemocyanins larger than a hexamer.

In conclusion, in this study we identified for the first time differences in the local environment of aromatic residues due to different conformations and conformation-dependent active site geometry. Information about active site geometry by XAS and spectroscopy of aromatic side chains (CD, fluorescence) revealed differences in these properties for particular conditions. In all cases investigated except one (oxygenated, XAS) hemocyanin structure differs if sample entrapped under deoxygenated and oxygenated are compared. Analysis of oxygen binding data based on the Nested-MWC-model resulted in conformational distributions for the different situations, which were not for all situations completely unambiguous (see ESI $\dagger$ ) but contained in each case a subset which is compatible with the structural information.

\section{Experimental}

\section{Protein purification}

Hemocyanins of Carcinus aestuarii was purified as previously described $^{53}$ and stored at $-20{ }^{\circ} \mathrm{C}$ in $50 \mathrm{mM}$ Tris buffer $\mathrm{pH} 7.5$ 
containing $20 \mathrm{mM} \mathrm{CaCl}_{2}, 20 \%$ sucrose. Before preparation of the samples, hemocyanin was dialyzed overnight against $50 \mathrm{mM}$ Tris buffer containing $20 \mathrm{mM} \mathrm{CaCl}_{2}$, glycerol $25 \%$ at the desired $\mathrm{pH}(6.5,7.5,7.8)$.

\section{Hemocyanin encapsulation}

Protein encapsulation in silica gels was carried out after a modified version of the protocol reported by Khan. ${ }^{32} \mathrm{~A}$ solution containing $125 \mu \mathrm{L}$ of tetramethyl orthosilicate (FLUKA SIGMA-ALDRICH, St. Louis, MO, 99\% pure) and $125 \mu \mathrm{L}$ of Tris $50 \mathrm{mM}, \mathrm{CaCl}_{2} 20 \mathrm{mM}, 25 \%$ glycerol (v/v) at the desired pH (6.5 or 7.8) was gently stirred on a vortexer for 30 seconds, then $70 \mu \mathrm{L}$ of hemocyanin at the desired concentration were added. After the gelification of a thin layer $(\sim 2 \mathrm{~mm})$ of sol-gel into a properly designed Teflon holder (see the ESI $\dagger$ ), the sample was covered with buffer and aged at $4{ }^{\circ} \mathrm{C}$ overnight. Samples were repeatedly rinsed with buffer before use. This step is required to remove the unreacted material and to equilibrate the sample with desired media as in the case of SANS measurements. The overall time between starting the gelification and an experimental session was consistently $48 \mathrm{~h}$ in order to normalize possible time dependent changes of sol-gel structure, as pointed out in previous work ${ }^{54,55}$ (see ESI, Table S1, Fig. S2 and S3†). Moreover, the time required for SANS/spectroscopic/functional characterization is negligible in comparison with the $48 \mathrm{~h}$ pre-aging time.

Deoxygenated samples were prepared under a pure nitrogen atmosphere in a glove-box. The UV-visible absorption spectrum was taken before and after each measurement and found to be essentially unaltered. The final concentration of hemocyanin was chosen according to the sensitivity of each biophysical technique used as specified in the relative paragraphs. Thus, the final concentration in the encapsulation mixture varied from $2 \mathrm{~g} \mathrm{~L}^{-1}$ (used for the optical spectroscopy measurements) to $5 \mathrm{~g} \mathrm{~L}^{-1}$ (XAS and SANS measurements). In order to avoid differences in the final composition of mixture which yield the sol-gel, the volumes of tetramethyl orthosilicate, buffer and hemocyanin solutions mentioned above were kept constant in all preparation. The different concentrations of hemocyanin reported were obtained by using different aliquots of a highly concentrated stock solution which could be diluted to a constant final volume.

Due to the dodecameric structure, hemocyanin has a molecular weight of $900 \mathrm{kDa}$. Thus, all experiments were carried out on sol-gel resulting from mixing $2.2-5.6 \mu \mathrm{M}$ protein with $2.6 \mathrm{M}$ alkoxide, 1.3 M glycerol and 19.5 mM Tris. The large (approx. $10^{6}$ fold) excess of silicate versus protein allows an homogenous distribution of protein molecules within the sol-gel.

\section{Spectroscopic measurements}

All spectroscopic measurements were carried out at $20{ }^{\circ} \mathrm{C}$ by inserting the samples in the Teflon holder into a quartz-cell filled with buffer Tris $50 \mathrm{mM}, \mathrm{CaCl}_{2} 20 \mathrm{mM}, 25 \%$ glycerol (v/v) at the desired $\mathrm{pH}$ and covered with a gas tight membrane. Absorption spectra of the samples were recorded with a HP 8452A diode-array spectrophotometer (HEWLETT PACKARD, Mississauga, Canada). Protein concentration was determined based on an absorption coefficient at $278 \mathrm{~nm}$ of $E_{278}^{0.1 \%}=1.24 \mathrm{~g}^{-1}$ $\mathrm{L} \mathrm{cm}{ }^{-1} \cdot{ }^{44}$ The absorbance ratio $A_{338} / A_{280}$, corrected for the background, for hemocyanin fully saturated with oxygen was 0.21 both in the sol-gel matrix and in solution. ${ }^{24}$ Circular dichroism spectra (CD) were recorded with a JASCO J-710 dichrograph (JASCO International Co, Tokyo, Japan). Samples were prepared at $0.4 \mathrm{~g} \mathrm{~L}^{-1}$ hemocyanin concentration for protein in solution (optical path length $10 \mathrm{~mm}$ ) and $2 \mathrm{~g} \mathrm{~L}^{-1}$ for sol-gel measurements (optical pathlength $2 \mathrm{~mm}$ ). Each spectrum was obtained by the average of 10 scans to minimize the signal-to-noise ratio and was converted to units of molar ellipticity after subtracting the spectrum of the empty sol-gel matrix in buffer solution. No further smoothing was applied to the data. Fluorescence spectra were recorded with a Perkin Elmer LS 50B spectrofluorimeter (PERKIN ELMER, Norwalk, CT) equipped with a thermostatic cell holder, excited at 278 or 295 $\mathrm{nm}$. Background intensities of empty sol-gel matrix in buffer were always subtracted from the samples. All spectra were normalized to the value at the emission maximum. Cu K-edge Xray absorption spectra of hemocyanin embedded into sol-gels were collected on beamline BM29 (now BM23) at the ESRF of Grenoble (France), using flat Si(111) crystal monochromator with an acquisition time of $5 \mathrm{~s}$ per scan point. Sol-gel matrices, prepared at $\mathrm{pH} 7.8$ and at $5 \mathrm{~g} \mathrm{~L}^{-1}$ hemocyanin concentration, were formed into the Teflon cell and inserted into the sample holder described in ESI. $\uparrow$ Then, the optical windows were closed with kapton films (DU PONT, Wilmington, DE) to avoid dehydration and filled with the buffer at $\mathrm{pH}$ 7.8. X-ray edge spectra were corrected for the pre- and post-edge regions of the spectrum. Spectra were recorded for the oxygenated forms of the different encapsulated conformers (Deoxy ${ }_{760}^{\mathrm{EM}}, \mathrm{Oxy}_{760}^{\mathrm{EM}}$ ) by equilibrating the bulk solution containing the protein-doped sol-gel with air. At this $\mathrm{pH}$, the oxygen affinity of encapsulated protein is high enough to saturate the protein to more than $90 \%$ at the oxygen partial pressure of air. The deoxygenated forms of the conformers (Deoxy ${ }_{0}^{\mathrm{EM}}, \mathrm{Oxy}_{0}^{\mathrm{EM}}$ ) were obtained by chemical consumption of dissolved oxygen by $10 \mathrm{mM}$ sodium dithionite (SIGMA-ALDRICH).

\section{Oxygen binding measurements}

Oxygen binding curves of C. aestuarii hemocyanin $\left(2 \mathrm{~g} \mathrm{~L}^{-1}\right)$ were obtained at $20^{\circ} \mathrm{C}$ employing a gas-mixing system (Model P-gas proportioner, AALBORD, Orangeburg, NY) and a spectrophotometer to collect the absorption spectra. The gas mixer was connected to the quartz cell through impermeable tubes and needles. By changing the relative fluxes of oxygen (analytical quality) and nitrogen (analytical quality), a specific oxygen partial pressure was adjusted and was transferred into the cell ( $\pm 5 \%$ of full scale accuracy). The degree of oxygenation of hemocyanin was followed via the change in $A_{338}$ corrected for the light scattering contribution quantified at $600 \mathrm{~nm}$. After each incremental step in oxygen concentration, the gas flow was kept constant until the $A_{338}$ has become constant, indicating equilibration of embedded hemocyanin with bulk oxygen through the sol-gel matrix. The relative saturation $Y$ at a given oxygen partial pressure $x$ (in Torr), $Y(x)$, was calculated from the 
absorbance at $338 \mathrm{~nm}(A(x))$ in relation to the absorbance in absence of oxygen $\left(A_{\mathrm{O}}\right)$ and under saturating conditions $\left(A_{\mathrm{S}}\right)$ as follows:

$$
Y(x)=\frac{A(x)-A_{\mathrm{O}}}{A_{\mathrm{s}}-A_{\mathrm{O}}}
$$

To obtain a measure for cooperativity $\left(n_{50}\right)$ and the oxygen partial pressure necessary to yield half-saturation $\left(p_{50}\right)$, oxygen binding data were plotted according to the Hill equation

$$
\log \frac{Y}{1-Y}=n_{\mathrm{H}} \log x-n_{\mathrm{H}} \log p_{50}
$$

where $x$ represents the oxygen partial pressure (in Torr) and $n_{\mathrm{H}}$ is the Hill coefficient. The slope at $\log \frac{Y}{1-Y}=0$ (where $x=p_{50}$ ) corresponds to $n_{50}$, the Hill-coefficient at $p_{50}$. The affinity for binding of the first and the last oxygen molecules was estimated from the asymptotes of the Hill plots. Here, the propagation of the experimental error introduces some uncertainty, thus these numbers were used only for a first estimation.

For a more detailed analysis, the binding data were analyzed in terms of the binding polynomials based on different models. Since the asymptotes in the Hill-plots suggested the existence of at least three different conformations, data were analyzed based on linear extensions of the two-state model. ${ }^{56}$ Here, an allosteric unit of size $n$ exists, which can adopt three or four different conformations ( $\mathrm{T}, \mathrm{S}$ and $\mathrm{R}$ or $\mathrm{W}, \mathrm{T}, \mathrm{S}$ and $\mathrm{R}$ ) with different oxygen binding affinities, yielding the following binding polynomial (eqn (3)):

$$
\begin{aligned}
P_{4 \text { state }}= & \left(1+K_{\mathrm{R}} x\right)^{n}+L_{\mathrm{S}}\left(1+K_{\mathrm{S}} x\right)^{n}+L_{\mathrm{T}}\left(1+K_{\mathrm{T}} x\right)^{n} \\
& +L_{\mathrm{W}}\left(1+K_{\mathrm{W}} x\right)^{n}
\end{aligned}
$$

where

$$
L_{\mathrm{S}}=\frac{\left[\mathrm{S}_{\mathrm{o}}\right]}{\left[\mathrm{R}_{\mathrm{o}}\right]}, \quad L_{\mathrm{T}}=\frac{\left[\mathrm{T}_{\mathrm{o}}\right]}{\left[\mathrm{R}_{\mathrm{o}}\right]}, \quad L_{\mathrm{W}}=\frac{\left[\mathrm{W}_{\mathrm{o}}\right]}{\left[\mathrm{R}_{\mathrm{o}}\right]}
$$

and $K_{\mathrm{R}}, K_{\mathrm{S}}, K_{\mathrm{T}}$, and $K_{\mathrm{W}}$ are the binding constants of the R-, S-, T-, and $\mathrm{W}$-states, respectively. The allosteric equilibrium constants $L_{\mathrm{S}}$ and $L_{\mathrm{T}}$ describe the ratio of the corresponding conformation relative to conformation $\mathrm{R}$ in the deoxygenated state. For $L_{\mathrm{S}}=$ $L_{\mathrm{W}}=0$ we obtain the MWC-model ${ }^{2}$ for $L_{\mathrm{W}}=0$ a three-state model. Alternatively, we employed the Nested-MWC-model as used before for hemocyanin of $C$. aestuarii, ${ }^{24}$ which assumes four different conformations (rR, tR, rT, tT) which are coupled in two levels of allosteric units. ${ }^{57}$ The binding polynomial for this case is given by eqn (4):

$$
P_{\text {nest }}=P_{\mathrm{R}}^{2}+\Lambda P_{\mathrm{T}}^{2}=\left(P_{\mathrm{rR}}^{n}+l_{\mathrm{R}} P_{\mathrm{tR}}^{n}\right)^{2}+\Lambda\left(P_{\mathrm{rT}}^{n}+l_{\mathrm{T}} P_{\mathrm{tT}}^{n}\right)^{2}
$$

where

$$
\begin{aligned}
& \Lambda=L \frac{\left(1+l_{\mathrm{R}}\right)^{2}}{\left(1+l_{\mathrm{T}}\right)^{2}} \\
& P_{\alpha \beta}=1+K_{\alpha \beta} x \\
& \alpha \beta=\mathrm{rR}, \mathrm{tR}, \mathrm{rT}, \mathrm{tT}
\end{aligned}
$$

In the data analysis the binding constants $K_{\alpha \beta}$ and allosteric equilibrium constants $l_{\mathrm{T}}, l_{\mathrm{R}}$ and $\Lambda$ were fitted to the data. We chose $\Lambda$ rather than $L$ as independent parameter, since this parameter directly reflects the ratio of $\mathrm{rT} / \mathrm{rR}$ molecules, whereas $L$ reflects $(\mathrm{tT}+\mathrm{rT}) /(\mathrm{tR}+\mathrm{rR})$, and thus $\Lambda$ is easier to interpret. The function describing the saturation degree $(\bar{x})$ is obtained from the binding polynomial $P$ by the following operation:

$$
\bar{x}=\frac{\partial \ln P}{n \partial \ln x}
$$

Allowing for some uncertainty in the completely unligated and the completely ligated form, the function fitted to the data reads (eqn (5)):

$$
f=a+b \bar{x}
$$

The value of $a$ was kept between -0.03 and +0.03 , and that of $b$ between 0.97 and 1.03 by appropriate constraints. For those data that did not show any positive cooperativity, a function describing a superposition of four hyperbolic binding curves was fitted to the data (eqn (6))

$$
\begin{aligned}
f= & a_{\mathrm{o}}+a_{\mathrm{tT}} \frac{K_{\mathrm{tT}} x}{1+K_{\mathrm{tT}} x}+a_{\mathrm{rT}} \frac{K_{\mathrm{rT}} x}{1+K_{\mathrm{rT}} x}+a_{\mathrm{tR}} \frac{K_{\mathrm{tR}} x}{1+K_{\mathrm{tR}} x} \\
& +a_{\mathrm{rR}} \frac{K_{\mathrm{rR}} x}{1+K_{\mathrm{rR}} x}
\end{aligned}
$$

with $a_{\mathrm{tT}}, a_{\mathrm{rT}}, a_{\mathrm{tR}}, a_{\mathrm{rR}}$ denoting the fraction of molecules in the corresponding conformation. The sum of all four was restricted to the range $0.97 \leq\left(a_{\mathrm{tT}}+a_{\mathrm{rT}}+a_{\mathrm{tR}}+a_{\mathrm{rR}}\right) \leq 1.03$ in the fitting routine. Data analysis was performed bases on the LevenbergMarquardt-routine built into to SigmaPlot 11 (SYSTAT SOFTWARE INC., San Jose, CA).

\section{Small-angle X-ray (SAXS) and neutron (SANS) scattering}

SAXS measurements of the sol-gel matrices without hemocyanin were performed at the ELETTRA Synchrotron Radiation facility (SAXS beamline, Trieste, Italy), in the $Q$-range from 0.019 to $0.46 \AA^{-1}$. Further details on SAXS experiments are given in ESI. $\dagger$ The curves were analyzed with the structure factor for fractal objects developed by Teixeira ${ }^{58}$ by means of the GENFIT software. ${ }^{59}$ Parameters of the model are the fractal dimension $D$, the characteristic radius $r_{0}$ of the silica repeat unit and the cutoff parameter $\xi$, which estimates the average dimension of the pores.

SANS curves of hemocyanins entrapped in sol-gel were collected at the ILL neutron source (D22 instrument, Grenoble, France) in the $Q$-range $0.012-0.2 \AA^{-1}$. In this experiment, all samples were prepared at the value of deuteration degree $\left(x_{\mathrm{D}}\right)$ corresponding to the matching point previously determined (see ESI $\dagger$ ). In these conditions, the SANS curves mainly contain information on the protein form factor $P(Q)$. The neutron scattering curves of entrapped hemocyanins were analyzed by the QUAFIT method, ${ }^{60,61}$ to find the optimum configuration of the dodecamer. Center coordinates and overall orientations of the single subunits forming the hexamer were fixed to the values found by previous SAXS measurements. ${ }^{23}$ The main 
QUAFIT fitting parameters of the protein form factor are: the Euler rotational angles $\alpha, \beta$ and $\gamma$ that define the orientation of one hexamer with respect to the other one; the distance $d$ between the centers of the two hexamers; the relative mass density $d_{\mathrm{w}}$ of hydration water. Moreover a scaling factor $\kappa$ and a constant background $B$ (which takes into account incoherent scattering effects) were optimized to fit the form factor provided by QUAFIT to the experimental SANS curves, ${ }^{62,63}$ according to eqn (7):

$$
\frac{\mathrm{d} \Sigma}{\mathrm{d} \Omega}(Q)=\kappa \frac{c N_{\mathrm{A}}}{M} P(Q)+B
$$

where $c$ is the w/v protein concentration, $N_{\mathrm{A}}$ Avogadro's number and $M$ the dodecamer molecular weight.

\section{Abbreviations}

MWC Monod-Wyman-Changeaux allosteric model

XAS X-ray absorption spectrum

Deoxy $^{\text {EM }}$ Hemocyanin embedded in sol-gel under deoxygenated conditions

$\mathrm{Oxy}^{\mathrm{EM}} \quad$ Hemocyanin embedded in sol-gel under oxygenated conditions

CD Circular dichroism

SAXS Small-angle X-ray scattering

SANS Small-angle neutron scattering

ITC Isothermal titration calorimetry

$\mathrm{SS}^{2} \quad$ Sum of squared residuals

$x_{\mathrm{D}} \quad$ Deuteration grade

\section{Acknowledgements}

We thank Olivier Mathon (XAS beamline BM29 at ESRF, Grenoble), Lionel Porcar (SANS instruments D22 and D11 at ILL, Grenoble) and Heinz Amenitsch (SAXS beamline at Elettra, Trieste) for their suggestions to improve XAS, SANS and SAXS measurements. We thank also Gerard Canters and Leandro Tabares (University of Leiden, The Netherlands) for their suggestions to optimize the application to our system of sol-gel methodology.

\section{References}

1 Q. Cui and M. Karplus, Protein Sci., 2008, 17, 1295-1307.

2 J. Monod, J. Wyman and J. P. Changeux, J. Mol. Biol., 1965, 12, 88-118.

3 M. F. Perutz, Nature, 1970, 228, 726-739.

4 A. Mozzarelli, C. Rivetti, G. L. Rossi, E. R. Henr and W. A. Eaton, Nature, 1991, 351, 416-419.

5 N. Shibayama and S. Saigo, J. Mol. Biol., 1995, 251, 203-209. 6 N. Shibayama and S. Saigo, FEBS Lett., 2001, 492, 50-53.

7 C. S. Viappiani, S. Bettati, L. Bruno, L. Ronda, S. Abbruzzetti, A. Mozzarelli and W. A. Eaton, Proc. Natl. Acad. Sci. U. S. A., 2004, 101, 14414-14419.

8 H. Decker, N. Hellmann, E. Jaenicke, B. Lieb, U. Meissner and J. Markl, Integr. Comp. Biol., 2007, 4, 631-644.
9 M. Beltramini, N. Colangelo, F. Giomi, L. Bubacco, P. Di Muro, N. Hellmann, E. Jaenicke and H. Decker, FEBS J., 2005, 272, 2060-2075.

10 M. A. Menze, N. Hellmann, H. Decker and M. K. Grieshaber, Biochemistry, 2005, 44, 10328-10338.

11 N. Hellmann, M. Paoli, F. Giomi and M. Beltramini, Arch. Biochem. Biophys., 2010, 495, 112-121.

12 H. Decker, P. R. Connelly, C. H. Robert and S. J. Gill, Biochemistry, 1988, 27, 6901-6908.

13 H. Decker and R. Sterner, J. Mol. Biol., 1990, 211, 281-283.

14 A. Volbeda and W. G. J. Hol, J. Mol. Biol., 1989, 209, 249-279.

15 B. Hazes, K. A. Magnus, C. Bonaventura, J. Bonaventura, Z. Dauter, K. H. Kalk and W. G. J. Hol, Protein Sci., 1993, 2, 597-619.

16 K. A. Magnus, B. Hazes, H. Ton-That, C. Bonaventura, J. Bonaventura and W. G. J. Hol, Proteins, 1994, 19, 302-309.

17 M. Metz and E. I. Solomon, J. Am. Chem. Soc., 2001, 123, 4938-4950.

18 J. Yoon, S. Fujii and E. I. Solomon, Proc. Natl. Acad. Sci. U. S. A., 2009, 106, 6585-6590.

19 E. I. Solomon, D. E. Heppner, E. M. Johnston, J. W. Ginsbach, J. Cirera, M. Qayyum, M. T. KieberEmmons, C. H. Kjaergaard, R. G. Hadt and L. Tian, Chem. Rev., 2014, 114, 3659-3853.

20 J. M. Brown, L. Powers, B. Kincaid, J. A. Larrabee and T. G. Spiro, J. Am. Chem. Soc., 1980, 102, 4210-4216.

21 N. J. Blackburn, R. W. Strange, J. Reedijk, A. Volbeda, A. Farooq, D. K. Kenneth and J. Zubieta, Inorg. Chem., 1989, 28, 1349-1357.

22 S. Hirota, T. Kawahara, M. Beltramini, P. Di Muro, R. S. Magliozzo, J. Peisach, L. S. Powers, N. Tanaka, S. Nagao and L. Bubacco, J. Biol. Chem., 2008, 283, 3194131948.

23 S. Hirota, N. Tanaka, I. Micetic, P. Di Muro, S. Nagao, H. Kitagishi, K. Kano, R. S. Magliozzo, J. Peisach, M. Beltramini and L. Bubacco, J. Biol. Chem., 2010, 285, 19338-19345.

24 E. Dainese, P. Di Muro, M. Beltramini, B. Salvato and H. Decker, Eur. J. Biochem., 1998, 256, 350-359.

25 I. Mičetić, C. Losasso, P. Di Muro, G. Tognon, P. Benedetti and M. Beltramini, J. Struct. Biol., 2010, 171, 1-10.

26 L. M. Ellerby, C. R. Nishida, S. A. Yamanaka, B. Dunn, J. S. Valentine and J. I. Zink, Science, 1992, 28, 1113-1115.

27 J. Livage, J. Y. Barreau, J. M. Da Costa and I. Desportes, Solgel Optics III, 1994, pp. 493-503.

28 E. J. A. Pope, J. Sol-Gel Sci. Technol., 1995, 4, 225-229.

29 B. C. Dave, B. Dunn, J. S. Valentine and J. L. Zink, ACS Symp. Ser., 1996, 622, 351-365.

30 R. B. Bhatia and J. Brinker, Chem. Mater., 2000, 12, 24342441.

31 K. Yokoyama and W. T. Yip, J. Phys. Chem. B, 2005, 24, 48164823.

32 I. Khan, C. F. Shannon, D. Dantsker, A. J. Friedman, J. PerezGonzales-de-Apodaca and J. M. Friedman, Biochemistry, 2000, 39, 16099-16109.

33 U. Samuni, C. J. Roche, D. Dantsker, L. J. Juszczak and J. M. Friedman, Biochemistry, 2006, 45, 2820-2835. 
34 S. V. Lepeshkevich, M. V. Parkhats, I. I. Stepuro and B. M. Dzhagarov, Biochim. Biophys. Acta, 2009, 1794, 18231830.

35 L. Ronda, S. Faggiano, S. Bettati, N. Hellmann, H. Decker, T. Weidenbach and A. Mozzarelli, Gene, 2007, 398, 202-207.

36 G. Zauner, M. Strianese, L. Bubacco, T. J. Aartsma, A. W. J. W. Tepper and G. W. Canters, Inorg. Chim. Acta, 2008, 361, 1116-1121.

37 M. Beltramini, L. Bubacco, B. Salvato, L. Casella, M. Gullotti and S. Garofani, Biochim. Biophys. Acta, 1992, 1120, 24-32.

38 W. Erker, R. Hubler and H. Decker, Biochim. Biophys. Acta, 2008, 1780, 1143-1147.

39 R. Favilla, M. Goldoni, P. Di Muro, B. Salvato and M. Beltramini, Biochim. Biophys. Acta, 2002, 1597, 42-50.

40 G. M. Luo, Q. Zhang, A. R. Del Castillo, V. Urban and H. O'Neil, ACS Appl. Mater. Interfaces, 2009, 10, 2262-2268.

41 C. G. Kalodimos, Ann. N. Y. Acad. Sci., 2012, 1260, 81-86.

42 W. A. Eaton, E. R. Henry, J. Hofrichter and A. Mozzarelli, Nat. Struct. Biol., 1999, 6, 351-358.

43 W. A. Eaton, E. R. Henry, J. Hofrichter, A. Mozzarelli, S. Bettati, C. Viappiani and A. Mozzarelli, Life, 2007, 59, 586-599.

44 S. Morris and C. R. Bridges, Integr. Comp. Biol., 1994, 34, 216-228.

45 N. Hellmann, IUBMB Life, 2011, 63, 329-336.

46 K. E. Wheeler, N. S. Lees, R. J. Gurbiel, S. L. Hatch, J. M. Nocek and B. M. Hoffman, J. Am. Chem. Soc., 2004, 126, 13459-13463.

47 M. A. García-Sanchez, V. De la Luz, M. L. Estrada-Rico, M. M. Murillo-Martínez, M. I. Coahuila-Hernández, R. Sosa-Fonseca, S. R. Tello-Solís, F. Rojas and A. Campero, J. Non-Cryst. Solids, 2009, 355, 120-125.

48 M. A. García-Sanchez, V. De la Luz, M. I. CoahuilaHernández, F. Rojas-González, S. R. Tello-Solís and A. Campero, J. Photochem. Photobiol., A, 2011, 223, 172-181.
49 I. N. Serratos, F. Rojas-González, R. Sosa-Fonseca, J. M. Esparza-Schulz, V. Campos-Peña, S. R. Tello-Solís and M. A. García-Sanchez, J. Photochem. Photobiol., A, 2013, 272, 28-40.

50 M. A. García-Sanchez, F. Rojas-González, C. MenchacaCampos, S. R. Tello-Solís, R. I. Y. Quiroz-Segoviano, L. A. Díaz-Alejo, E. Salas-Bañales and A. Campero, Molecules, 2013, 18, 588-653.

51 R. I. Y. Quiroz-Segoviano, I. N. Serratos, F. Rojas-González, S. R. Tello-Solís, R. Sosa-Fonseca, O. Medina-Juárez, C. Menchaca-Campos and M. A. García-Sanchez, Molecules, 2014, 19, 2261-2285.

52 E. Salas-Bañales, R. I. Y. Quiroz-Segoviano, L. A. Díaz-Alejo, F. Rojas-González, A. Estrella-González, A. Campero and M. A. García-Sanchez, Molecules, 2015, 20, 19463-19488.

53 L. Bubacco, R. S. Magliozzo, M. Beltramini, B. Salvato and J. Peisach, Biochemistry, 1992, 31, 9294-9303.

54 C. J. Brinker and G. W. Scherer, J. Non-Cryst. Solids, 1985, 70, 301-322.

55 J. Dumas, J. F. Quinson, C. Bovier, S. Baza and J. Serughetti, J. Non-Cryst. Solids, 1986, 82, 220-224.

56 A. P. Minton and K. Imai, Proc. Natl. Acad. Sci. U. S. A., 1974, 71, 1418-1421.

57 C. H. Robert, Biopolymers, 1990, 30, 335-347.

58 J. Teixeira, J. Appl. Crystallogr., 1988, 21, 781-785.

59 F. Spinozzi, C. Ferrero, M. G. Ortore, A. De Maria Antolinos and P. Mariani, J. Appl. Crystallogr., 2014, 47, 1132-1139.

60 F. Spinozzi and M. Beltramini, Biophys. J., 2012, 103, 511521.

61 F. Spinozzi, P. Mariani, I. Micetic, C. Ferrero, D. Pontoni and M. Beltramini, PLoS One, 2012, 7, e49644, DOI: 10.1371/ journal.pone.0049644.

62 C. D. Putman, M. Hammel, G. L. Hura and J. A. Tainer, Q. Rev. Biophys., 2007, 40, 191-285.

63 H. Stuhrmann, Rep. Prog. Phys., 2004, 67, 1073-1115. 